\title{
Proteasome inhibitor MG132 suppresses pancreatic ductal adenocarcinoma-cell migration by increasing ESE3 expression
}

\author{
FANJIE JIN $^{1}$, DI XIAO ${ }^{2}$, TIANSUO ZHAO ${ }^{1}$ and MING YU ${ }^{2}$ \\ ${ }^{1}$ Department of Pancreatic Cancer, Tianjin Medical University Cancer Institute and Hospital, \\ National Clinical Research Center for Cancer, Key Laboratory of Cancer Prevention and Therapy, Tianjin 300060; \\ ${ }^{2}$ Department of Nutrition and Food Science, School of Public Health, Tianjin Medical University, Tianjin 300070, P.R. China
}

Received February 11, 2019; Accepted September 27, 2019

DOI: $10.3892 / \mathrm{ol} .2019 .11157$

\begin{abstract}
The clinical significance of the proteasome inhibitor MG132 has been examined in numerous human cancer types; however, its influence on the metastasis and progression of pancreatic cancer is yet to be determined. In the present study, the effect of MG132 treatment on pancreatic ductal adenocarcinoma (PDAC) cell lines (SW1990 and PANC-1) was examined. Compared with the control groups, MG132 treatment resulted in higher expression levels of ETS homologous factor (ESE3), a crucial member of the E26 transformation-specific family that is central to various differentiation and development processes in epithelial tissues. MG132 treatment also increased the nuclear translocation of ESE3. Mechanistically, MG132 further inhibited the invasion and migration of PDAC cells by promoting E-cadherin expression, which not only plays an important role in cell-cell adhesion, but is also a direct target of ESE3. Furthermore, subsequent knockdown experiments, using short interfering RNAs, demonstrated that MG132 upregulated E-cadherin via an increase in ESE3 expression. The results of the present study support the hypothesis that MG132 treatment inhibits PDAC metastasis, highlighting the potential of MG132 as a therapeutic agent for the treatment of patients with PDAC.
\end{abstract}

\section{Introduction}

Pancreatic ductal adenocarcinoma (PDAC) exhibits a high propensity for metastasis, and is therefore considered to be a highly lethal human malignancy $(1,2)$ with a low 5-year survival rate of $<5 \%$ (3-6). Previous reports have demonstrated

Correspondence to: Dr Tiansuo Zhao, Department of Pancreatic Cancer, Tianjin Medical University Cancer Institute and Hospital, National Clinical Research Center for Cancer, Key Laboratory of Cancer Prevention and Therapy, 7F Huanhuxi Road, Tianjin 300060, P.R. China

E-mail: jfj_tjmuch@aliyun.com

Key words: pancreatic ductal adenocarcinoma, MG132, PANC-1, ETS homologous factor, E-cadherin that both transcriptional and translational downregulation of E-cadherin in parental cells initiated epithelial-mesenchymal transition (EMT), consequently promoting metastasis (7-9). The inhibition of adhesion between epithelial cells (cell-cell adhesion) is known to be an essential step during the early stage of tumor metastasis, and typically involves the downregulation of E-cadherin expression $(10,11)$.

The ubiquitin-proteasome pathway is associated with the degradation of regulatory proteins that influence DNA repair, signal transduction and apoptosis, and therefore, the proteasome represents a promising target for cancer therapy $(12,13)$. MG132 is a well-characterized proteasome inhibitor that induces caspase-8-dependent osteosarcoma-cell apoptosis (14). Moreover, MG132 was reported to inhibit the transcriptional activity and expression of the oncogenic transcription factor forkhead box M1, promoting apoptosis in several human cancer types (15). Similarly, the anti-proliferative effect of MG132 and cisplatin co-administration for the treatment of osteosarcoma xenograft models was superior to the monotherapeutic effect of cisplatin, supporting the potential of MG132/cisplatin for the treatment of patients with osteosarcoma (12). Additionally, it was discovered that MG132 may upregulate the expression of nuclear factor erythroid 2-related factor 2 and increase proteasome activity via the inhibition of diabetic nephropathy in an animal model (16). Collectively, the aforementioned results suggest that MG132 affects the growth of numerous solid tumor types, and should consequently be considered as a therapeutic target.

E26 transformation-specific family transcription factor (ESE3) plays a pivotal role in the differentiation and development programs of numerous epithelial tissue types. ESE3 has been reported to bind directly to its target genes with the propensity to regulate EMT, stem-like physiological processes (an increased ability to form spheres, similar to epithelial stem cells) (17-19) and tumor progression (17,20). Therefore, ESE3 is considered as a potential tumor suppressor gene associated with pancreatic cancer. In addition, ESE3 was shown to inhibit the migration and invasion capacities of PDAC cells (both in vitro and in vivo), suggesting that the mechanism behind its regulation of PDAC metastasis may involve the regulation of E-cadherin expression levels at the transcriptional level (21). In summary, the aim of the present study was to investigate the effect of MG132 on the expression level of E-cadherin 
and the accumulation of ESE3, and to determine its influence on the migration and invasion abilities of PDAC cells.

\section{Materials and methods}

Reagents and short interfering (si)RNAs. Proteasome inhibitor MG132 (cat. no. SML1135) and cycloheximide (CHX; cat. no. C104450) were purchased from Sigma-Aldrich; Merck KGaA. The primary antibodies were purchased from Abcam and include; ESE3 (cat. no. ab24337), E-cadherin (cat. no. ab76055) and $\beta$-actin (cat. no. E4D9Z; all Cell Signaling Technology, Inc.) antibodies. To determine the influence of ESE3 expression levels on MG132 activity, ESE3 was knocked down in PANC-1 and SW1990 cells by the transfection of specific siRNAs (ESE3\#1 forward, 5'-GCCAGUGGCAUG AAAUUCATT-3' and reverse, 5'-UGAAUUUCAUGCCAC UGGCTT-3'; and ESE3\#2 forward, 5'-CAGCCGAGCUAU GAGAUAUTT-3' and reverse, 5'-AUAUCUCAUAGCUCG GCUGTT-3') and an unrelated silencing sequence was synthesized as a negative control (siNC forward, 5'-1UUCUCCGA ACGUGUCACGUTT-3' and reverse, 5'-ACGUGACACGUUC GGAGAATT-3'). All siRNAs were purchased from Sangon Biotech Co., Ltd. Stock solutions of MG132 were prepared in DMSO at a concentration of $10 \mathrm{mg} / \mathrm{ml}$ and then diluted in cell culture medium to a final working concentration of $10 \mu \mathrm{M}$.

Cell culture conditions and pharmacological treatment. The SW1990 and PANC-1 PDAC cell lines were cultured in Dulbecco's modified Eagle's medium (DMEM; Gibco; Thermo Fisher Scientific, Inc.) containing $10 \%$ fetal bovine serum (FBS, Shanghai ExCell Biology, Inc.) at $37^{\circ} \mathrm{C}$, in a humidified culture chamber with $5 \% \mathrm{CO}_{2}$. The cells were detached from the culture plates using trypsin $(0.05 \%)$ and EDTA $(0.5 \mathrm{mM})$ in phosphate-buffered saline (PBS). The cell lines were treated with $10 \mu \mathrm{M}$ MG132 for $0,2,4$ and $6 \mathrm{~h}$ at $37^{\circ} \mathrm{C}$. Groups of control cells treated with DMSO only were evaluated in parallel to the experimental groups. Cycloheximide (CHX), a protein-synthesis inhibitor, was used to test the degradation of proteins following MG132 and DMSO treatment at $50 \mu \mathrm{g} / \mathrm{ml}$ for $4 \mathrm{~h}$. All experiments were repeated three times.

Inhibitory effects of MG132. The half-maximal inhibitory concentration $\left(\mathrm{IC}_{50}\right)$ of MG132 on PDAC cells was detected using the Real-Time Cellular Analysis (RTCA) system (ACEA Biosciences, Inc.). DMEM (10\% FBS) was added to the E-Plate assay (ACEA Biosciences, Inc.) to determine the background impedance values. Log-phase cells were collected and counted to achieve a suspension of $4 \times 10^{3}$ cells/well; the cells were then added to the E-Plate on a test stand (having been previously incubated at $37^{\circ} \mathrm{C}$ and $5 \% \mathrm{CO}_{2}$ ), and left to react at room temperature for $30 \mathrm{~min}$. Real-time dynamic cell-proliferation detection was performed overnight at $37^{\circ} \mathrm{C}, 5 \% \mathrm{CO}_{2}$. The MG132 stock solution was added to the corresponding concentration gradient solutions, and real-time dynamic detection was continued. The cell effect curves and $\mathrm{IC}_{50}$ values were obtained using the RTCA Software (RTCA Software Lite 2.2.1).

Western blotting. The cells were washed with PBS and then lysed using RIPA buffer containing $1 \%$ protease inhibitor (both
Sigma-Aldrich; Merck KGaA). Equal amounts of protein were then resolved using 10\% SDS-PAGE. Protein concentrations were quantified using the Pierce protein assay kit (cat. no. UA269551, Thermo Fisher Scientific, Inc.). The separated proteins (20 $\mu \mathrm{g} / \mathrm{lane})$ were carefully transferred to polyvinylidene fluoride membranes at $4^{\circ} \mathrm{C}$ for $2 \mathrm{~h}$. Subsequently the proteins were blocked using $5 \%$ skimmed milk (BD Biosciences) and probed with the following primary antibodies: Anti-ESE3 (1:1,000; cat. no. ab24337), anti-E-cadherin (1:1,000; both Abcam; cat. no. ab76055) and $\beta$-actin (1:5,000; cat. no. E4D9Z; Cell Signaling Technology, Inc.) for $2 \mathrm{~h}$ at room temperature. Following primary incubation, the membranes were incubated with horseradish peroxidase-conjugated goat anti-rabbit (1:10,000; ProteinTech Group, Inc; cat. no. SA00001-2)/anti-mouse secondary antibodies (1:10,000; ProteinTech Group, Inc; cat. no. SA00001-1) for $1 \mathrm{~h}$ at room temperature. Protein quantification was performed using Image Lab Software (version 5.2.1, Bio-Rad Laboratories, Inc).

Wound-healing and invasion assays. For the wound-healing assay, the PANC-1 and SW1990 cells (treated with either MG132 or DMSO) were seeded into a 24 -well plate prior to wounding. The wounds were created by scratching across the monolayer using sterile pipette tips. The cells were induced with DMEM containing 1\% FBS. After a 48-h incubation in a humidified culture chamber $\left(5 \% \mathrm{CO}_{2}\right)$ the cells were imaged using an inverted routine microscope (magnification, $\mathrm{x} 40$; ECLIPSE Ts2; Nikon Corporation).

For the invasion assay, $1 \times 10^{5}$ cells were added to the upper chamber of a transwell insert with DMEM containing $1 \% \mathrm{FBS}$, and filters coated with Matrigel (1:5 dilution, BD Biosciences) were used. DMEM containing $10 \%$ FBS was added to the lower chamber and used as a chemoattractant. The cells were subsequently incubated under controlled conditions $\left(37^{\circ} \mathrm{C}\right.$ and $5 \% \mathrm{CO}_{2}$ ) for $18 \mathrm{~h}$. A three-step stain set (Corning Inc.) was used to stain the cells that had migrated to the bottoms of the filters at room temperature (each step was stained for $5 \mathrm{~min}$ ). Then, 10 different fields of view were randomly selected, and cell counting was performed using an inverted routine microscope (magnification, 40x; ECLIPSE Ts2; Nikon Corporation). The mean cell numbers were calculated and statistical analyses were performed on these values.

Immunofluorescence. To investigate the expression patterns of ESE3 in PDAC cells treated with MG132, 2x10 3 PANC-1 cells were seeded onto glass microscope slides, washed with PBS, and then fixed with $4 \%$ paraformaldehyde for $10 \mathrm{~min}$ at room temperature. The cells were then permeabilized with $0.3 \%$ Triton X-100 in PBS for 5 min at room temperature. To minimize non-specific staining, the cells were treated with $3 \%$ bovine serum albumin (Sigma-Aldrich; Merck KGaA) in PBS for $1 \mathrm{~h}$. During the immunolabeling step, an anti-ESE3 antibody (1:100 dilution) was used to stain the cells, overnight at $4^{\circ} \mathrm{C}$. The nuclei of the labeled cells were subsequently stained with 4',6-diamidino-2-phenylindole (DAPI) Fluoromount-G medium (Southern Biotech), and the slides were viewed using a confocal fluorescence microscope (magnification, x200; LSN 880; ZEISS; GmbH).

Statistical analysis. All statistical analyses were performed using GraphPad Prism 7.0 software (GraphPad Software, Inc.). 
A

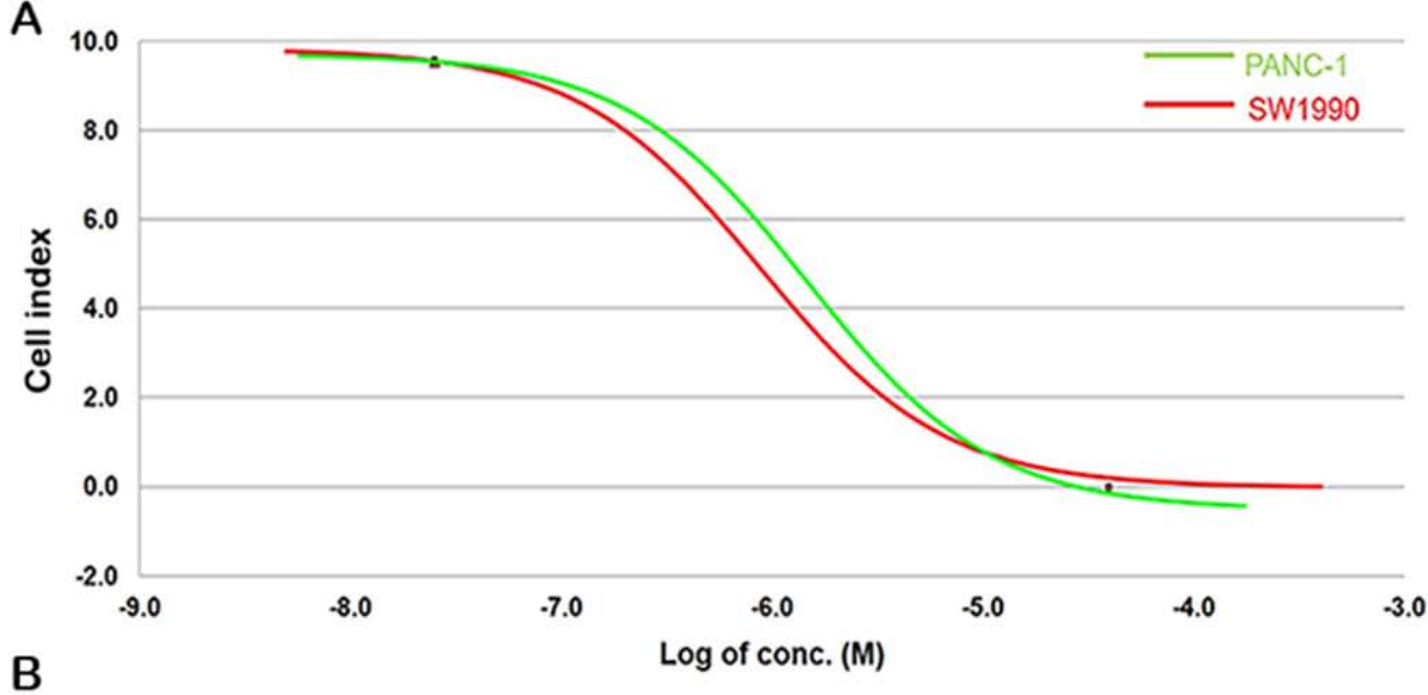

B

DMSO

MG132(10 $\mu \mathrm{M})$
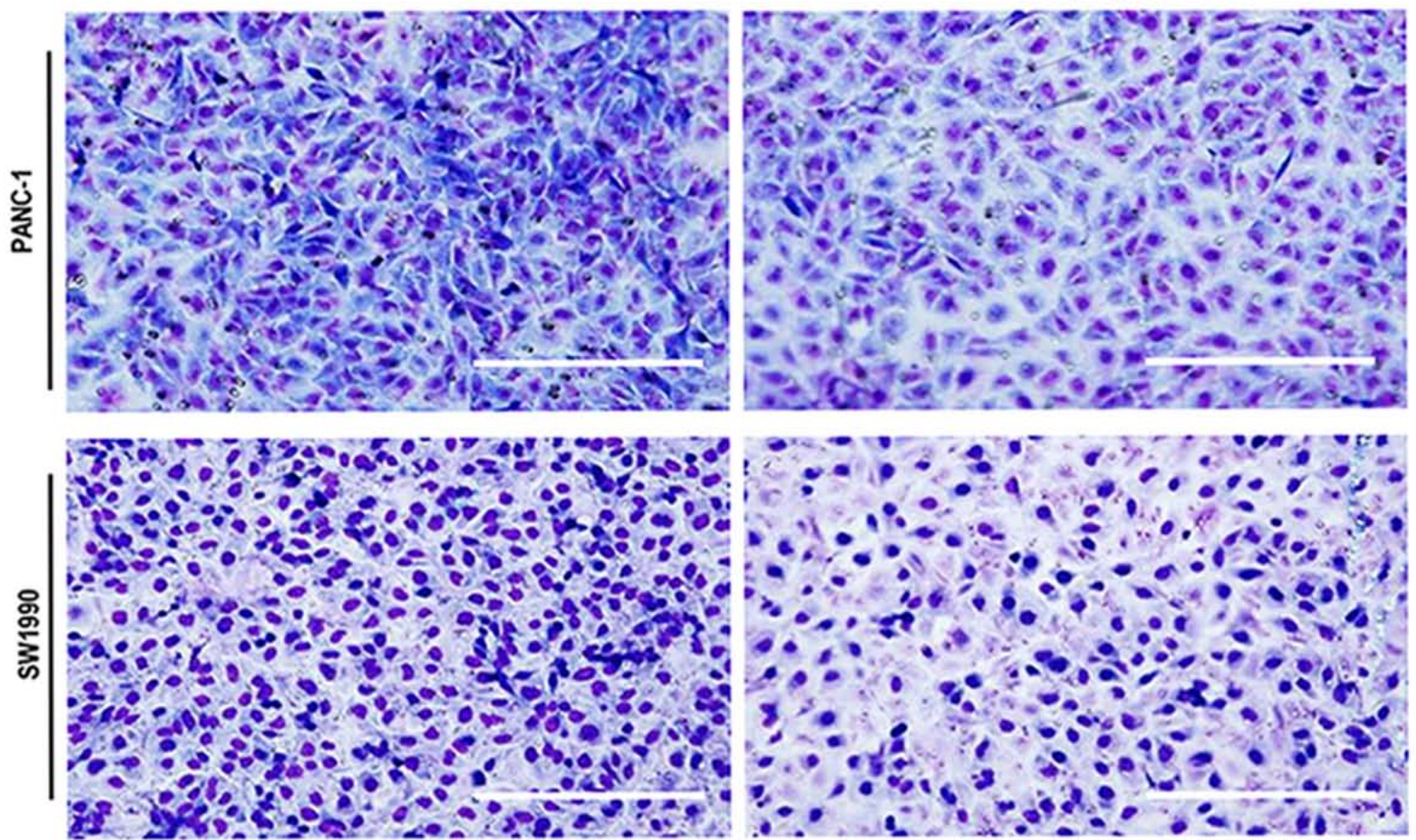

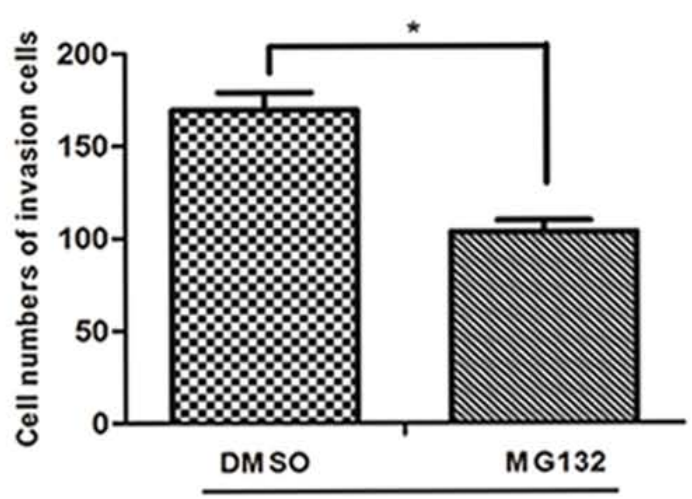

PANC-1

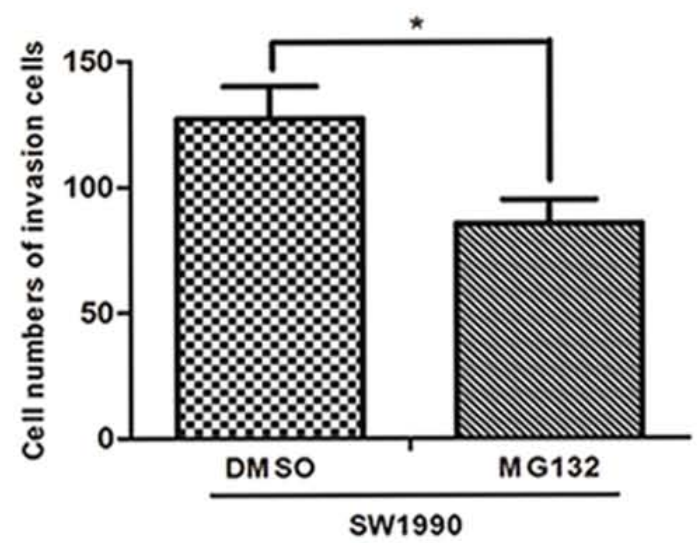

Figure 1. Proteasome inhibitor MG132 exhibits cytotoxic effects and inhibits the migration and invasion of pancreatic cancer cells. (A) Following the treatment of PANC-1 and SW1990 cells for $48 \mathrm{~h}$ at various concentrations of MG132 $(0.25-20 \mu \mathrm{M})$, IC $\mathrm{I}_{50}$ curves were plotted using real-time cell analysis. (B) Migration of PANC-1 and SW1990 cells $18 \mathrm{~h}$ post-treatment with MG132. Invasive cells were counted using a three-step staining process (DMSO-treated group and $10 \mu \mathrm{M}$ MG132-treated group). All experiments were repeated three times. ${ }^{*} \mathrm{P}<0.05$ vs. the control group. 


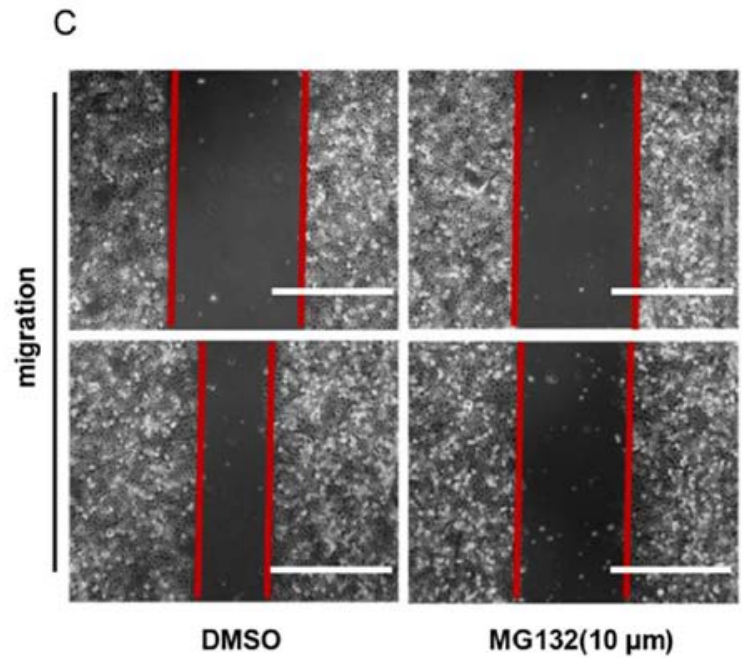

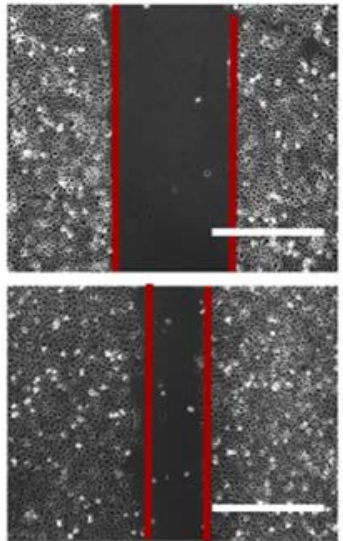

DMSO
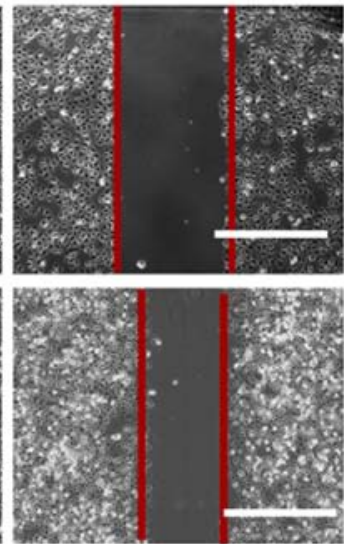

MG132(10 $\mu \mathrm{m})$
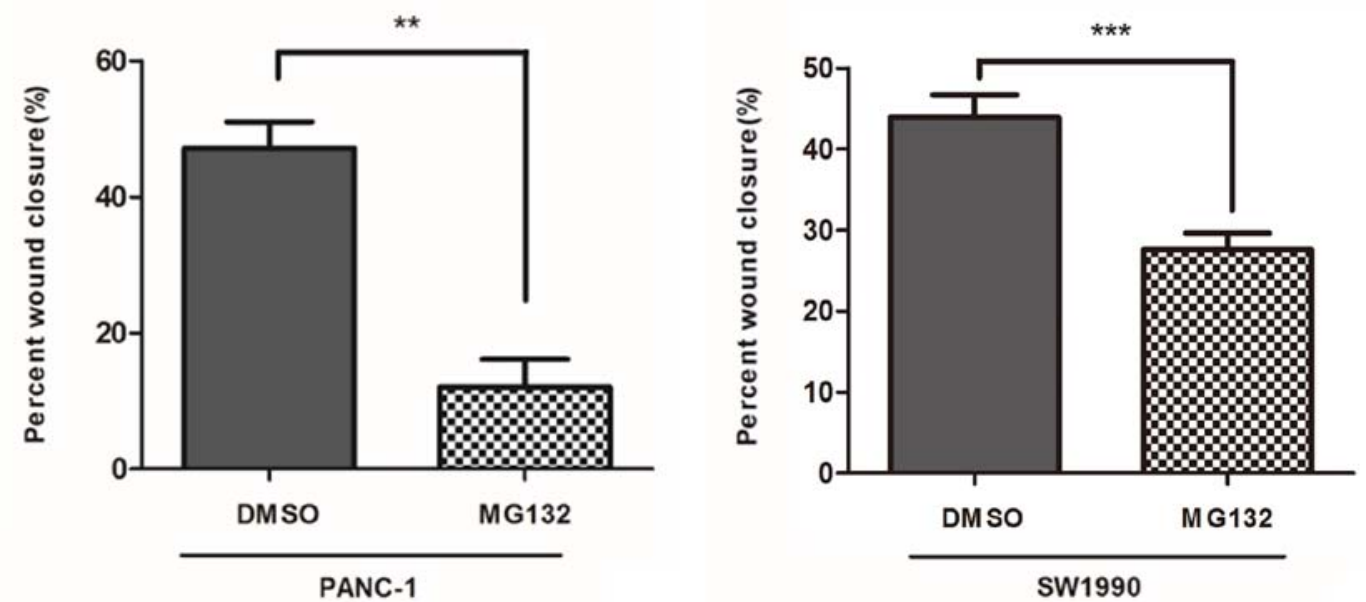

Figure 1. Continued. (C) Wound-healing assay showing the differences in PANC-1 and SW1990 cell motility 0 (top) and 48 h (bottom) post-treatment with MG132. The results and representative images are shown for PANC-1 and SW1990 cells treated with or without MG132. The bar chart represents the migration index for each treatment. Values were calculated relative to the wound-closure distance, compared with that of the DMSO group. ${ }^{* *} \mathrm{P}<0.01$ and ${ }^{* * * *} \mathrm{P}<0.001$. Scale bar, $200 \mu \mathrm{m}$. $\mathrm{IC}_{50}$, half-maximal inhibitory concentration.

The unpaired Student's t-test or Mann-Whitney U test were used to compare the differences between two groups, and one-way ANOVA followed by the SNK-q test was used to compare $\geq 3$ groups. The results are presented as the mean \pm standard error of the mean (SEM) and $\mathrm{P}<0.05$ was considered to indicate a statistically significant result.

\section{Results}

Proteasome inhibitor MG132 exhibits cytotoxic effects and inhibits the migration and invasion of pancreatic cancer cells. After $48 \mathrm{~h}$ of treatment, the $\mathrm{IC}_{50}$ values of MG132 in PANC-1 and SW1990 PDAC cells were $11.20 \pm 0.742$ and $11.18 \pm 0.787 \mu \mathrm{M}$, respectively, according to RTCA (Fig. 1A). Based on this finding, and consistent with previously reported literature (22), the MG132 concentration was fixed at $10 \mu \mathrm{M}$ for subsequent experiments.

The effect of MG132 on the invasion cell number of PANC-1 and SW1990 cells was evaluated using Transwell chambers. The number of PANC-1 and SW1990 cells passing through the polycarbonate membrane at $18 \mathrm{~h}$ was reduced by $10 \mu \mathrm{M}$ MG132, compared with that of the DMSO-treated control group (Fig. 1B). The mean number of invading PANC-1 cells was 105 for the treatment group, compared with 169 for the control group, and the mean number of invading SW1990 cells was 85 for the treatment group, compared with 128 for the control group $(\mathrm{P}<0.05)$. The wound-healing assay revealed that the migration of PANC-1 and SW1990 cells was significantly reduced following MG132 treatment. The wound areas for PANC-1 cells were $\sim 12.0 \pm 4.1 \%$ at $48 \mathrm{~h}$ for the MG132-treated group, compared with $\sim 47.2 \pm 3.8 \%$ for the control group ( $\mathrm{P}<0.01$; Fig. 1C). SW1990-cell wound healing was also significantly suppressed, with wound areas of $227.6 \pm 2.0 \%$ at $48 \mathrm{~h}$ for the MG132-treated group, compared with $\sim 43.9 \pm 2.8 \%$ for the control group ( $\mathrm{P}<0.005$; Fig. 1C). The results of the present study suggest that MG132 is able to inhibit the invasion and migration properties of PANC-1 and SW1990 cells.

Degradation of ESE3 via the ubiquitin-proteasome pathway is inhibited by MG132. To investigate the potential mechanism of MG132-associated suppression of PDAC cell invasion and migration, western blotting and immunofluorescence experiments were performed. Following the treatment of pancreatic cell lines (PANC-1 and SW1990) with $10 \mu \mathrm{M}$ 
A

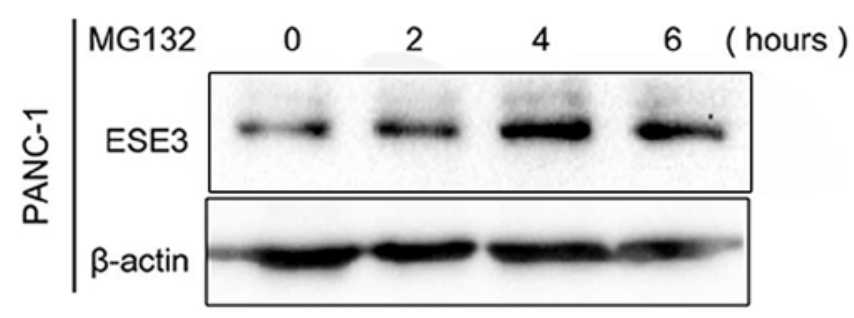

B



C



D

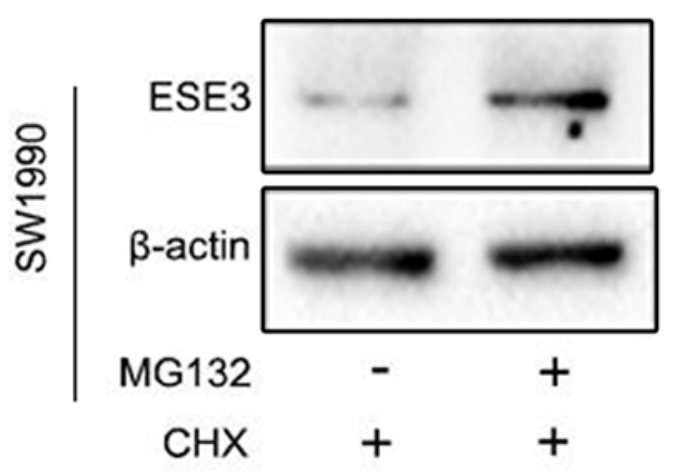

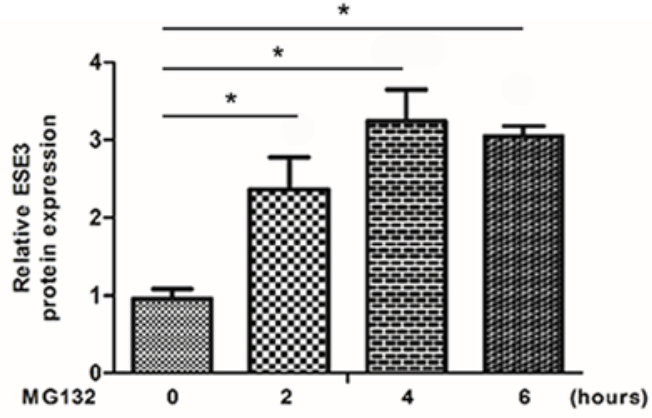
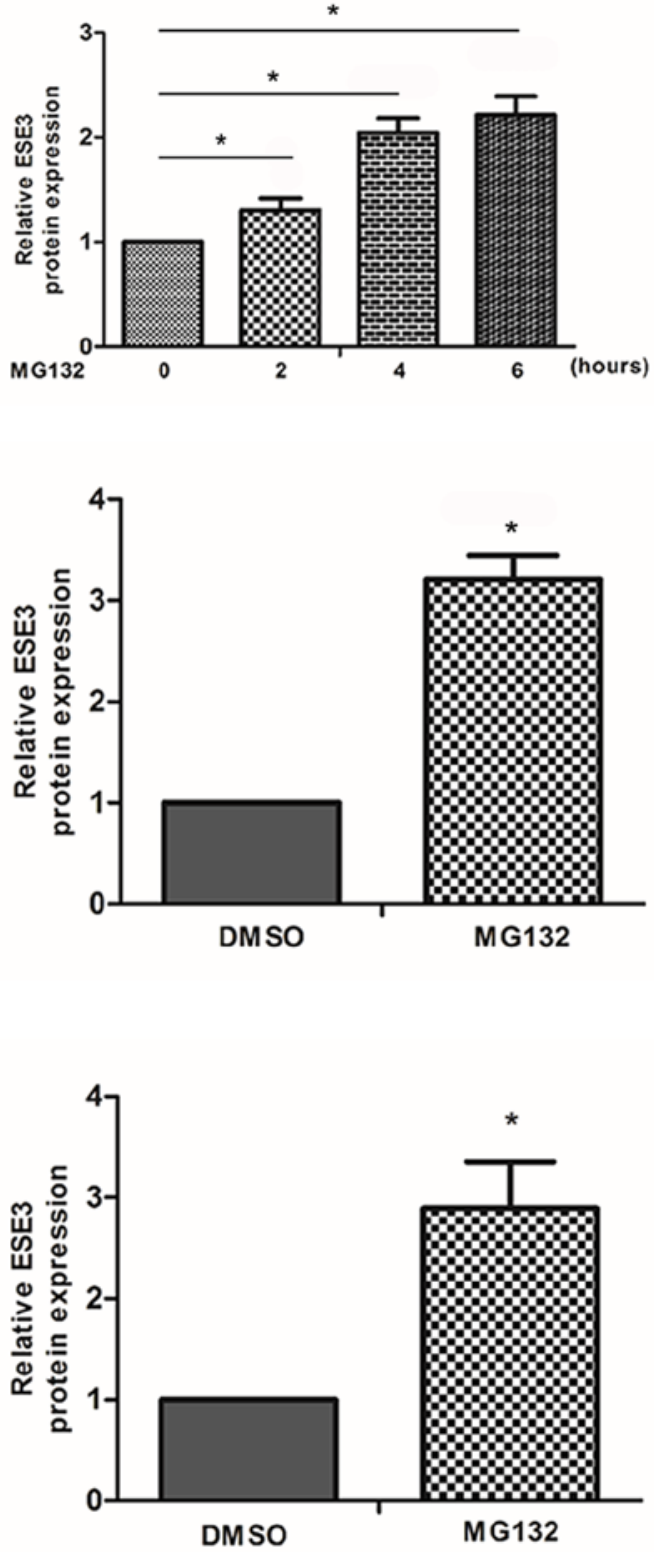

Figure 2. MG132 inhibits ESE3 degradation. Proteasome inhibition following MG132 treatment increased in a time-dependent manner in (A) PANC-1 and (B) SW1990 cells, resulting in increased accumulation of ESE3. ESE3 accumulation resulting from the co-treatment of (C) PANC-1 and (D) SW1990 cells with MG132 and/or CHX. Results are presented as the percentage \pm SEM compared with untreated control values from three independent experiments. ${ }^{*}<0.05$. ESE3, ETS homologous factor; CHX, cycloheximide.

MG132 for various time periods (0-6 h), the ESE3 expression levels increased in a time-dependent manner (Fig. 2A and B; $\mathrm{P}<0.05)$. To determine whether the observed increase involved an MG132-dependent pathway, $10 \mu \mathrm{M}$ MG132 was added to PANC-1 and SW1990 cells for $2 \mathrm{~h}$, followed by treatment with
CHX $(50 \mu \mathrm{g} / \mathrm{ml})$ for $4 \mathrm{~h}$ to inhibit ESE3 protein synthesis. Western blotting determined that ESE3 exhibited high expression levels following MG132 treatment (Fig. $2 \mathrm{C}$ and D; P<0.05). Thus, it was demonstrated that MG132 increased ESE3 levels via the inhibition of its proteasomal degradation. 
A
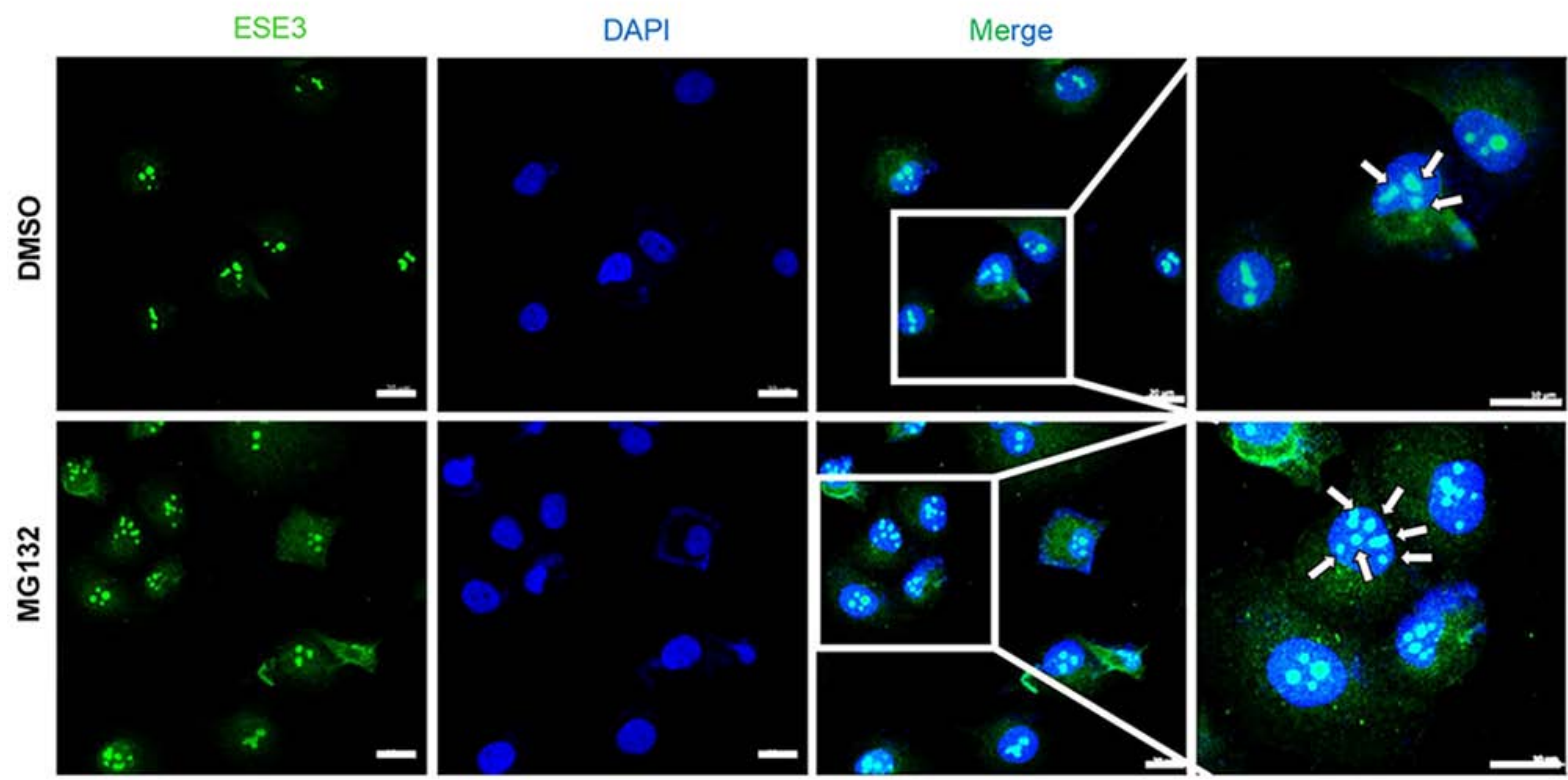

B



Figure 3. MG132 promotes ESE3 nuclear translocation. The cellular localization of endogenous ESE3 was examined using fluorescence microscopy in human PANC-1 cells treated with $10 \mu \mathrm{M}$ MG132 for $6 \mathrm{~h}$, and stained for ESE3 (green). Nuclear staining with DAPI is indicated in blue. Arrowheads indicate nuclear expression of ESE3. (A) Nuclear translocation of ESE3 increased in PANC-1 cells, following treatment with MG132. All experiments were repeated three times. (B) Relative expression levels of ESE3. "P<0.05. Magnification, 200x; scale bar=20 $\mu \mathrm{m}$. ESE3, ETS homologous factor.

MG132 increases ESE3 nuclear translocation. Previous studies have determined that ESE3 is primarily localized in the nucleus of normal epithelial esophageal cells $(20,23,24)$. Hence, the nuclear expression of ESE3 was investigated using immunofluorescence in PDAC cells treated with MG132. Fluorescent staining was performed to visualize the expression of ESE3 (Fig. 3A). Arrowheads indicate the nuclear expression of ESE3 (Fig. 3A), and the number of arrowheads was determined and compared with the DMSO-treated control group. The nuclear translocation of ESE3 was increased in PANC-1 cells treated with MG132 (Fig. 3A; P<0.05), yet no significant increase was observed in SW1990 cells.

MG132 upregulates E-cadherin expression levels via the upregulation of ESE3. The results of the present study indicated that treatment with MG132 was associated with increased expression of ESE3 in PDAC cells. To investigate the factors affecting the migration and invasion of PDAC cells down-stream of ESE3, E-cadherin [a classical EMT marker and a direct target of ESE3; (21)] expression levels were determined to be increased in both SW1990 and PANC-1 cell lines, following MG132 treatment (Fig. 4A and B). However, similar changes were not detected in other EMT-related markers such as N-cadherin or Snail. Additionally, in PANC-1 cells transfected with siESE3, the upregulation of E-cadherin expression caused by MG132 was significantly inhibited (Fig. 4C; $\mathrm{P}<0.05$ ). This suggested that MG132 directly stimulates E-cadherin expression via the increased expression of ESE3.

ESE3-knockdown and low E-cadherin expression reverse the inhibitory effect of MG132 on the migration and invasion abilities of PANC-1 and SW1990 cells. To assess the influence of ESE3 expression on the migration and invasion capacities of PANC-1 cells treated with MG132, ESE3 expression was knocked down using siESE3 and the effect was analyzed using Transwell and wound-healing assays. The results showed that knockdown of ESE3 expression significantly reduced the inhibitory effect of MG132 on PANC-1 and SW1990 cell 
A

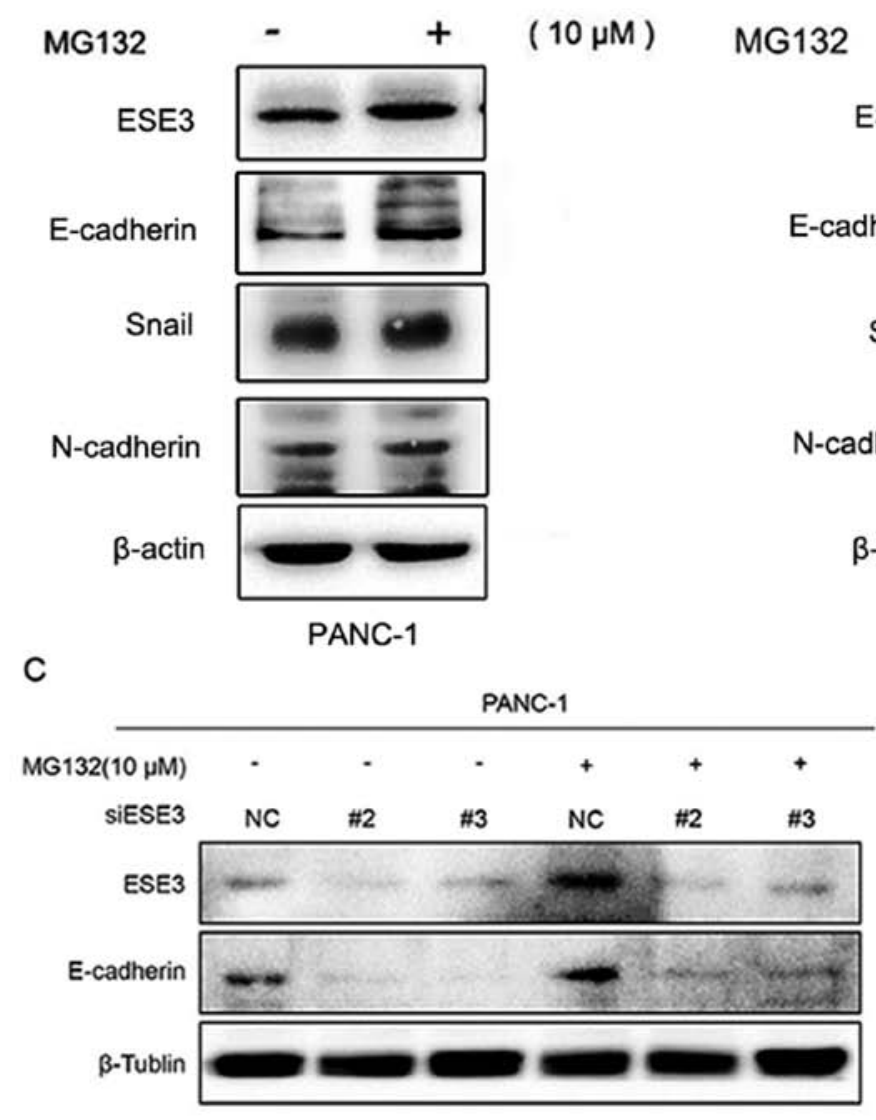

B



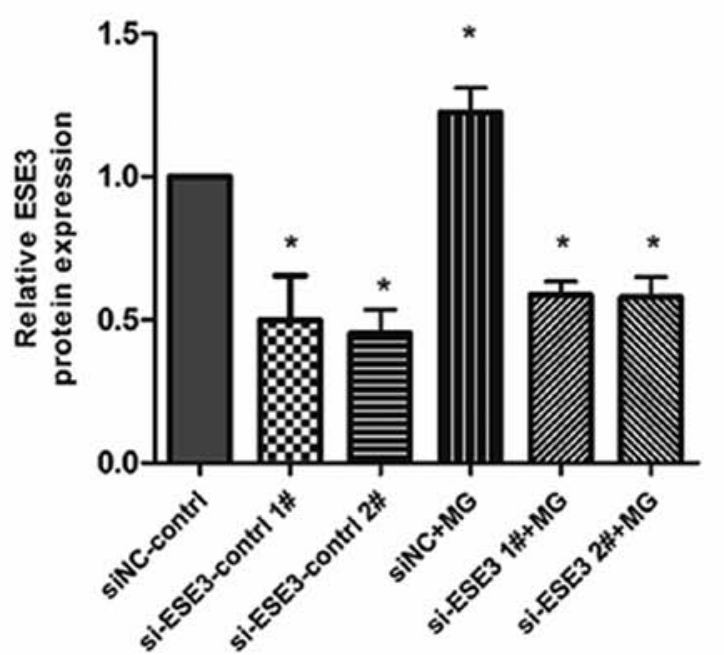

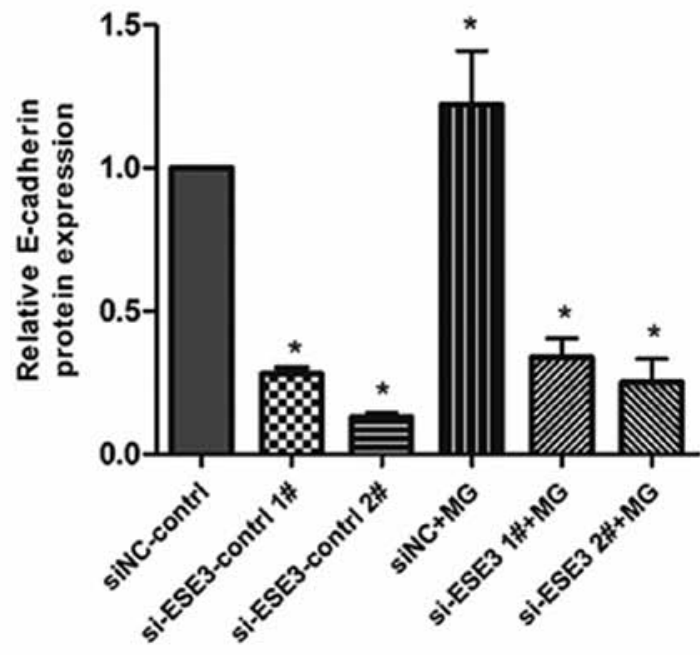

Figure 4. MG132 promotes E-cadherin expression via the upregulation of ESE3 expression. Treatment of (A) PANC-1 and (B) SW1990 cells with MG132 increased the expression levels of ESE3 and E-cadherin. No significant changes were detected in the expression of other EMT-related markers, including N-cadherin and Snail (vs. the DMSO group). PANC-1 cells were transfected with siRNAs specific to the ESE3 gene. (C) The effect of MG132 was blocked for the expression of both ESE3 and E-cadherin at the protein level. The data are presented as the mean value \pm SEM of arbitrary densitometric units (A.U.) or as the percentage \pm SEM of untreated control values from three independent experiments. ${ }^{*} \mathrm{P}<0.05$. ESE3, ETS homologous factor; siNC, short interfering negative control; MG, MG132.

invasiveness (Fig. 5A and B) and migration (Fig. 5C and D). These findings suggest that ESE3 is an important target under MG132 treatment conditions.

\section{Discussion}

It has been reported that during the early stages of tumor metastasis, it is important to inhibit cell-cell adhesion between epithelial cells (25-27). Indeed, inhibition of the cellular adhesion mediated by E-cadherin during this period represents a major step in the treatment of primary tumors, and serves as a classical EMT marker (28-30). As a nuclear factor, ESE3 may play a role in this process by directly binding to the promoter of the E-cadherin gene in PDAC cells, thereby stimulating its expression (21).

In the current study, it was determined that the proteasome inhibitor MG132 inhibited PDAC cell invasion and 
A

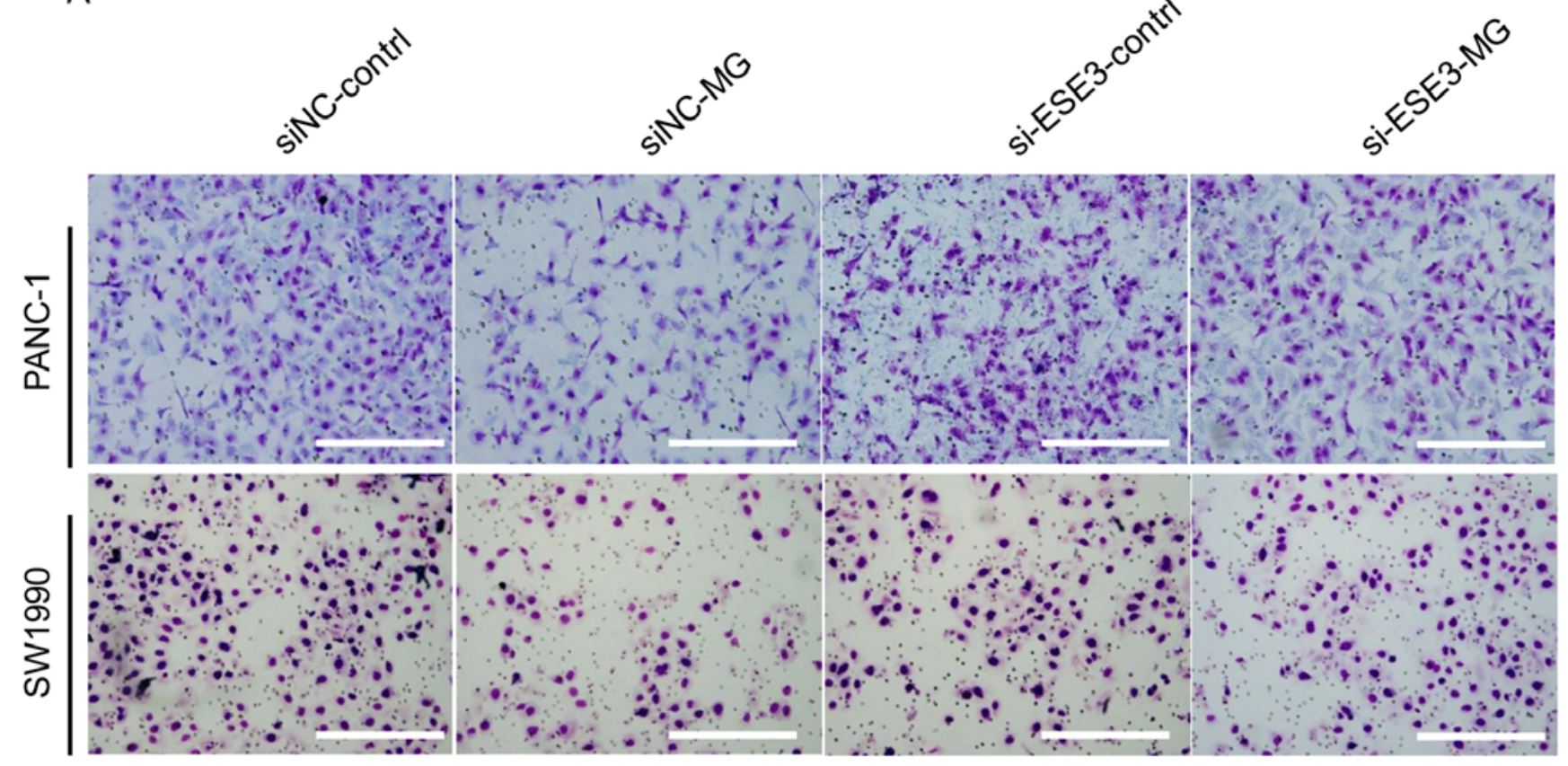

B
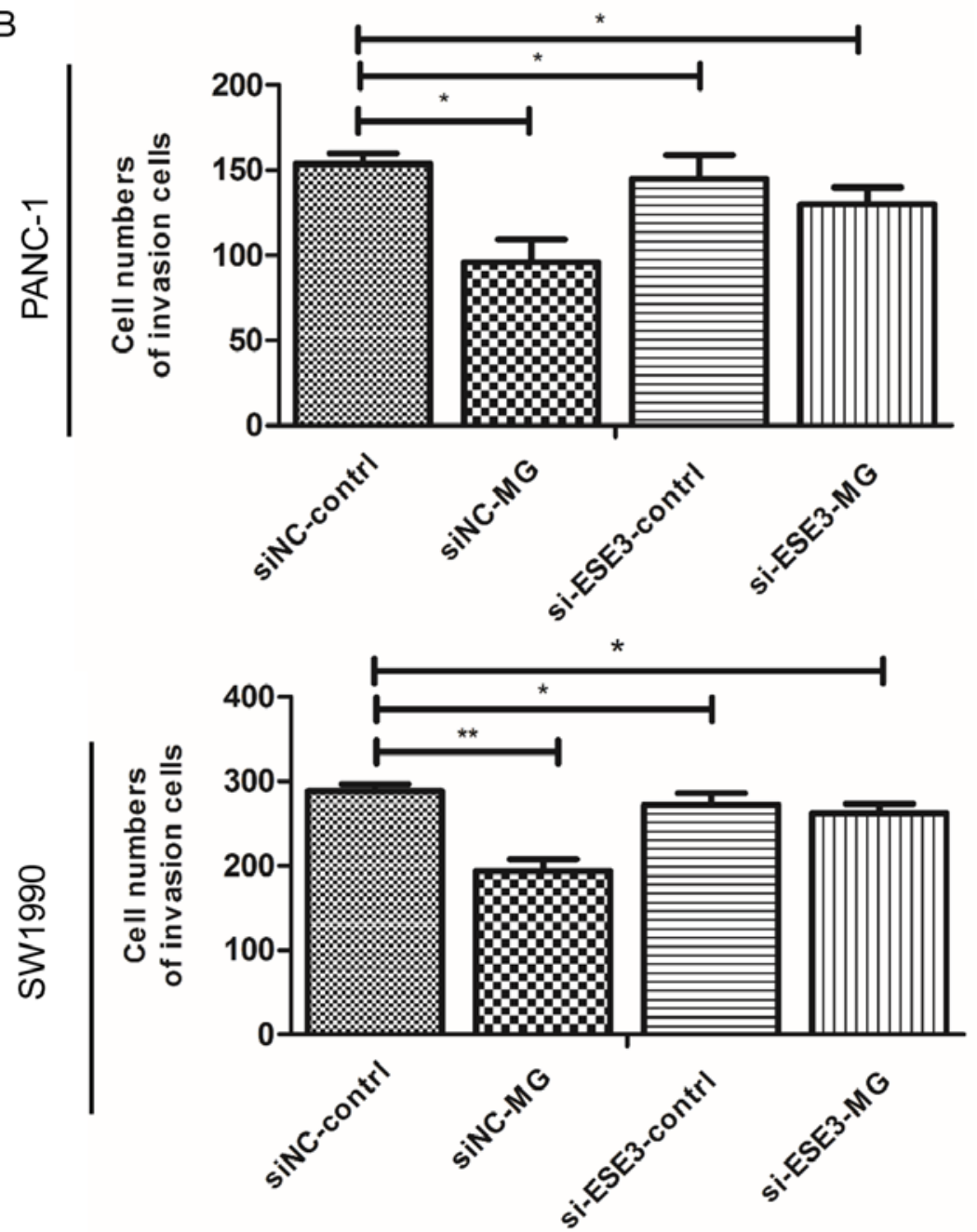

Figure 5. Knockdown of ESE3 and the expression level of E-cadherin inhibits the migration and invasion of PANC-1 cells. PANC-1 cells were transfected with preselected siRNAs targeting ESE3. (A) Invasion was inhibited in both the si-ESE3-control and si-ESE3-MG groups with or without MG132 treatment $(10 \mu \mathrm{M}, 48 \mathrm{~h})$, respectively. Results and representative images are exhibited for PANC-1 and SW1990 cells treated in the presence and absence of MG132. (B) Number of cells that flowed through the Transwell chamber. Bars represent the migration index of each treatment. ${ }^{*} \mathrm{P}<0.05$ and ${ }^{* *} \mathrm{P}<0.01$ vs. the control group. All experiments were repeated three times; scale bar, $200 \mu \mathrm{m}$. ESE3, ETS homologous factor; siNC, short interfering negative control; MG, MG132. 
C



D


Figure 5. Continued. Knockdown of ESE3 and the expression level of E-cadherin inhibits the migration and invasion of PANC-1 cells. PANC-1 cells were transfected with preselected siRNAs targeting ESE3. (C) Migration was inhibited in both the si-ESE3-control and si-ESE3-MG groups with or without MG132 treatment $(10 \mu \mathrm{M}, 48 \mathrm{~h})$, respectively. Results and representative images are exhibited for PANC-1 and SW1990 cells treated in the presence and absence of MG132. (D) Values were calculated relative to the closure distance of the cell monolayers. " $\mathrm{P}<0.05$ and ${ }^{* *} \mathrm{P}<0.01$ vs. the control group. All experiments were repeated three times; scale bar, $200 \mu \mathrm{m}$. ESE3, ETS homologous factor; siNC, short interfering negative control; MG, MG132. 
migration in vitro, which has not been previously reported. To determine the molecular mechanism underlying this process, the expression level of the EMT marker E-cadherin (but not snail or N-cadherin) was evaluated in PDAC cells. E-cadherin expression increased following treatment with MG132. Further investigation indicated that MG132 increased the accumulation of ESE3 by inhibiting its proteasomal degradation. An immunofluorescence assay was performed to investigate MG132-associated promotion of translocation to the nucleus in PANC-1 cells; however, no significant increase was observed in SW1990 cells. Data from the present study indicate that MG132 activated E-cadherin by influencing both the expression level and translocation of ESE3. The addition of CHX demonstrated that the increase in ESE3 levels was not influenced by an increase in protein synthesis. Thus, MG132 suppressed the invasion and migration of two PDAC cell lines by increasing ESE3 expression levels, via the suppression of the relevant proteasome pathway, and its down-stream target, the E-cadherin gene.

Subsequently, the central role of ESE3 in the inhibition of migration and invasion in PDAC cells (which was increased by MG132) was further investigated. ESE3 was knocked down by transfection of PDAC cells with siESE3. The rescue experiment demonstrated that siESE3 reduced MG132-induced inhibition of migration and invasion in PDAC cell lines. This conclusion was further supported by the discovery that MG132 promoted E-cadherin-induced PDAC migration and invasion through the accumulation of ESE3.

Future studies should focus on discovering the specific signaling pathway by which MG132 influences the expression of ESE3, as this may further explain the difference in translocation of ESE3 to the nucleus between PANC-1 cells and SW1990 cells, following MG132 treatment.

Overall, the findings of the present study improve our understanding of the biological function of MG132 in PDAC metastasis. The current results suggest that MG132 may be combined with other chemotherapeutic drugs for the treatment PDAC, in order to block the signaling pathway that downregulates ESE3 expression. By inhibiting PDAC metastasis, this strategy may result in improved patient outcome.

\section{Acknowledgements}

Not applicable.

\section{Funding}

The present was supported by Major Anticancer Technologies R\&D Program of Tianjin (grant. no. 12ZCDZSY16700) and the National Science Foundation of China (grant nos. 81502067, 31470951, 81302082,81272685, 31301151, 81172355, 31471340, 31470957, 81472264 and 81401957).

\section{Availability of data and material}

The datasets used and/or analyzed during the present study are available from the corresponding author on reasonable request.

\section{Authors' contributions}

FJ, TZ and MY conceived and designed the research. The experiments were performed by FJ and DX The data analysis was performed by FJ, DX, TZ and MY. MY and TZ contributed materials, reagents and analysis tools and aquired funding. The manuscript was written by FJ, DX ,TZ and MY. TZ and MY revised the manuscript . TZ and MY supervised the project.

\section{Ethics approval and consent to participate}

Not applicable.

\section{Patient consent for publication}

Not applicable.

\section{Competing interests}

The authors declare that they have no competing interests.

\section{References}

1. Roy R, Zurakowski D, Wischhusen J, Frauenhoffer C, Hooshmand S, Kulke M and Moses MA: Urinary TIMP-1 and MMP-2 levels detect the presence of pancreatic malignancies. Br J Cancer 111: 1772-1779, 2014.

2. Roe JS, Hwang CI, Somerville TDD, Milazzo JP, Lee EJ, Da Silva B, Maiorino L, Tiriac H, Young CM, MiyabayashiK, et al: Enhancer reprogramming promotes pancreatic cancer metastasis. Cell 170: 875-888.e20, 2017.

3. Vincent A, Herman J, Schulick R, Hruban RH and Goggins M: Pancreatic cancer. Lancet 378: 607-620, 2011

4. Knudsen ES, O'Reilly EM, Brody JR and Witkiewicz AK: Genetic diversity of pancreatic ductal adenocarcinoma and opportunities for precision medicine. Gastroenterology 150: 48-63, 2016.

5. Huang C, Li N, Li Z, Chang A, Chen Y, Zhao T, Li Y, Wang X, Zhang W, Wang Z, et al: Tumour-derived Interleukin 35 promotes pancreatic ductal adenocarcinoma cell extravasation and metastasis by inducing ICAM1 expression. Nat Commun 8: 14035, 2017.

6. DeSantis CE, Miller KD, Goding Sauer A, Jemal A and Siegel RL: Cancer statistics for African Americans, 2019. CA Cancer J Clin 69: 211-233, 2019.

7. Kotiyal S and Bhattacharya S: Events of molecular changes in epithelial-mesenchymal transition. Crit Rev Eukaryot Gene Expr 26: 163-171, 2016.

8. Amawi H, Ashby CR, Samuel T, Peraman R and Tiwari AK: Polyphenolic nutrients in cancer chemoprevention and metastasis: Role of the epithelial-to-mesenchymal (EMT) pathway. Nutrients 9: pii: E911, 2017.

9. Mao XY, Li QQ, Gao YF, Zhou HH, Liu ZQ and Jin WL: Gap junction as an intercellular glue: Emerging roles in cancer EMT and metastasis. Cancer Lett 381: 133-137, 2016.

10. Petit CS, Barreau F, Besnier L, Gandille P, Riveau B, Chateau D, Roy M, Berrebi D, Svrcek M, Cardot P, et al: Requirement of cellular prion protein for intestinal barrier function and mislocalization in patients with inflammatory bowel disease. Gastroenterology 143: 122-132.e15, 2012.

11. Moirangthem A, Bondhopadhyay B, Mukherjee M, Bandyopadhyay A, Mukherjee N, Konar K, Bhattacharya S and Basu A: Simultaneous knockdown of uPA and MMP9 can reduce breast cancer progression by increasing cell-cell adhesion and modulating EMT genes. Sci Rep 6: 21903, 2016.

12. Sun F, Zhang Y, Xu L, Li S, Chen X, Zhang L, Wu Y and Li J: Proteasome inhibitor MG132 enhances cisplatin-induced apoptosis in osteosarcoma cells and inhibits tumor growth. Oncol Res 26: 655-664, 2018.

13. Crawford LJ and Irvine AE: Targeting the ubiquitin proteasome system in haematological malignancies. Blood Rev 27: 297-304, 2013. 
14. Yan XB, Yang DS, Gao X, Feng J, Shi ZL and Ye Z: Caspase-8 dependent osteosarcoma cell apoptosis induced by proteasome inhibitor MG132. Cell Biol Int 31: 1136-1143, 2007.

15. Gartel AL: A new target for proteasome inhibitors: FoxM1. Expert Opin Investig Drugs 19: 235-242, 2010.

16. Cui W, Bai Y,Luo P, Miao L and Cai L: Preventive and therapeutic effects of MG132 by activating Nrf2-ARE signaling pathway on oxidative stress-induced cardiovascular and renal injury. Oxid Med Cell Longev 2013: 306073, 2013.

17. Albino D, Longoni N, Curti L, Mello-Grand M, Pinton S Civenni G, Thalmann G, D'Ambrosio G, Sarti M, Sessa F, et al: ESE3/EHF controls epithelial cell differentiation and its loss leads to prostate tumors with mesenchymal and stem-like features. Cancer Res 72: 2889-2900, 2012.

18. Longoni N, Kunderfranco P, Pellini S, Albino D, Mello-Grand M, Pinton S, D'Ambrosio G, Sarti M, Sessa F, Chiorino G, et al: Aberrant expression of the neuronal-specific protein DCDC2 promotes malignant phenotypes and is associated with prostate cancer progression. Oncogene 32: 2315-2324, 2324.e1-e4, 2013.

19. Albino D, Civenni G, Rossi S, Mitra A, Catapano CV and Carbone GM: The ETS factor ESE3/EHF represses IL-6 preventing STAT3 activation and expansion of the prostate cancer stem-like compartment. Oncotarget 7: 76756-76768, 2016

20. Wang L, Xing J, Cheng R, Shao Y, Li P, Zhu S and Zhang S: Abnormal localization and tumor suppressor function of epithelial tissue-specific transcription factor ESE3 in esophageal squamous cell carcinoma. PLoS One 10: e0126319, 2015.

21. Zhao T, Jiang W, Wang X, Wang H, Zheng C, Li Y, Sun Y, Huang C Han ZB, Yang S, et al: ESE3 inhibits pancreatic cancer metastasis by upregulating E-cadherin. Cancer Res 77: 874-885, 2017.

22. He M, Qiao Z, Wang Y, Kuai Q, Li C, Wang Y, Jiang X, Wang X, $\mathrm{Li} \mathrm{W}, \mathrm{He} \mathrm{M}$, et al: chidamide inhibits aerobic metabolism to induce pancreatic cancer cell growth arrest by promoting Mcl-1 degradation. PLoS One 11: e0166896, 2016.

23. Seth A and Watson DK: ETS transcription factors and their emerging roles in human cancer. Eur J Cancer 41: 2462-2478, 2005 .
24. Jain N, Morgan CE, Rife BD, Salemi M and Tolbert BS: Solution structure of the HIV-1 intron splicing silencer and its interactions with the UP1 domain of heterogeneous nuclear ribonucleoprotein (hnRNP) A1. J Biol Chem 291: 2331-2344, 2016.

25. Kahlert UD, Joseph JV and Kruyt FAE: EMT- and MET-related processes in nonepithelial tumors: Importance for disease progression, prognosis, and therapeutic opportunities. Mol Oncol 11: 860-877, 2017.

26. Kim KS, Kim J, Oh N, Kim MY and Park KS: ELK3-GATA3 axis modulates MDA-MB-231 metastasis by regulating cell-cell adhesion-related genes. Biochem Biophys Res Commun 498: 509-515, 2018.

27. Zhang M, Hu C, Tong D, Xiang S, Williams K, Bai W, Li GM, Bepler G and Zhang X: Ubiquitin-specific peptidase 10 (USP10) deubiquitinates and stabilizes MutS homolog 2 (MSH2) to regulate cellular sensitivity to DNA damage. J Biol Chem 291: 10783-10791, 2016

28. Sanchez-Tillo E, Lazaro A, Torrent R, Cuatrecasas M, Vaquero EC, Castells A, Engel P and Postigo A: ZEB1 represses E-cadherin and induces an EMT by recruiting the SWI/SNF chromatin-remodeling protein BRG1. Oncogene 29: 3490-3500, 2010.

29. Matos ML, Lapyckyj L, Rosso M, Besso MJ, Mencucci MV, Briggiler CI, Giustina S, Furlong LI and Vazquez-Levin MH: Identification of a novel human E-cadherin splice variant and assessment of its effects upon EMT-related events. J Cell Physiol 232: 1368-1386, 2017.

30. Feldkoren B, Hutchinson R, Rapoport Y, Mahajan A and Margulis V: Integrin signaling potentiates transforming growth factor-beta 1 (TGF- $\beta 1$ ) dependent down-regulation of E-cadherin expression-important implications for epithelial to mesenchymal transition (EMT) in renal cell carcinoma. Exp Cell Res 355: 57-66, 2017.

This work is licensed under a Creative Commons Attribution-NonCommercial-NoDerivatives 4.0 International (CC BY-NC-ND 4.0) License. 